\title{
LA PLANIFICACIÓN PARTICIPATIVA EN COTACACHI ¿ESPACIO ESTATAL O CIUDADANO? (1996-2018)
}

AUTORES: César Gonzalo Alvear Morales ${ }^{1}$

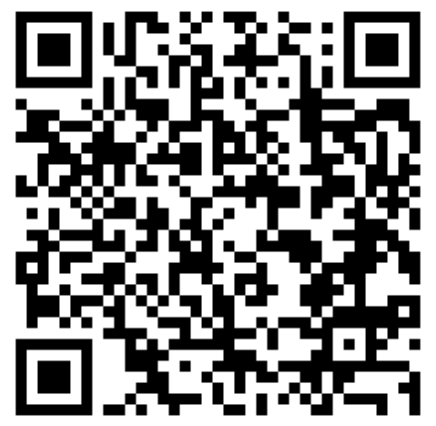

DIRECCIÓN PARA CORRESPONDENCIA:cesaralvear@yahoo.com

Fecha de recepción: 04/01/2020

Fecha de aceptación: 08/0272020

RESUMEN

La institucionalización de la planificación participativa local establecida por la Constitución de 2008, produjo en Cotacachi -referente nacional de la participación- un escenario de pugna en torno a los nuevos espacios de participación en la planificación, provocando un enfrentamiento entre la legalidad del gobierno local, establecido como autoridad estatal sobre la planificación participativa; y la legitimidad de los actores sociales, quienes durante una década y media antes de la Constitución, impulsaron y sostuvieron el proceso participativo en el cantón, tornándose en una autoridades no estatales de la participación. El presente estudio pretende dar cuenta de las posibilidades de acción política de los espacios de participación insertados en la planificación que, desde el gobierno local se implementaron como mecanismos de exclusión de actores no alineados a su bandera política; y desde los actores sociales se abordaron como espacios de acción organizativa en demanda de sus derechos de participación. Metodológicamente, mediante una investigación cualitativa se elaboró un estudio de caso que, bajo el enfoque analítico de la gubernamentalidad de Michel Foucault da a conocer las diversas estrategias de gobierno implementadas desde el Estado (central y local) y la sociedad civil por regir los espacios de participación en la planificación local. Llegando a concluir que el gobierno del actual régimen de planificación participativa en Cotacachi se produce bajo el influjo de diversas prácticas de poder basadas en conocimiento y autoridad implementadas por los actores estatales y sociales, logrando estos últimos, validar su actoría a través de capacidades técnicas especializadas y capital político organizacional.

\footnotetext{
${ }^{1}$ Ing. Agroindustrial, Msc en Desarrollo Local y Territorial, Investigador Independiente
} 
César Gonzalo Alvear Morales, , ...

PALABRAS CLAVE: Participación social, gobierno local, planificación local, gubernamentalidad.

\section{PARTICIPATORY PLANNING IN COTACACHI, ¿STATE SPACE OR CITIZENS SPACE? (1996-2018)}

\section{ABSTRACT}

The institutionalization of local participatory planning established by the 2008 Constitution, produced in Cotacachi -the national benchmark for participationa scenario of conflict over new spaces for participation in planning, causing a confrontation between the legality of local government, established as a state authority on participatory planning; and the legitimacy of the social actors, who for a decade and a half before the Constitution, promoted and sustained the participatory process in the canton, becoming non-state authorities for participation. The present study tries to give an account of the possibilities of political action of the spaces of participation inserted in the planning that, from the local government, were implemented as mechanisms of exclusion of actors not aligned to their political flag; and from the social actors they were approached as spaces for organizational action in demand of their participation rights. Methodologically, through a qualitative research, a case study was prepared that, under the analytical approach of the governmentality of Michel Foucault, reveals the various government strategies implemented by the State (central and local) and civil society for ruling the spaces of participation in local planning. Coming to conclude that the government of the current participatory planning regime in Cotacachi is produced under the influence of various power practices based on knowledge and authority implemented by state and social actors, the latter succeeding in validating their performance through specialized technical capacities. and organizational political capital.

KEYWORDS: Social participation, local government, local planning, governmentality.

\section{INTRODUCCIÓN}

La presente investigación se origina de un estudio de mayor magnitud orientado al análisis de la participación en ciudadana bajo el régimen de la Constitución de 2008 en el cantón Cotacachi, el cual ha sido uno de los más importantes referentes de planificación participativa en el país. Este estudio surgió con el interés de generar aportes para el análisis de la planificación participativa local instituida por la Carta Magna, al visibilizar las posibilidades de acción política que se pueden generar alrededor los espacios de participación en el proceso de planificación local, tanto desde el gobierno local como autoridad legal sobre los espacios de participación, así como desde la sociedad civil en su empoderamiento de la participación y sus capacidades desarrolladas para el ejercicio de sus derechos de participación.

A nivel latinoamericano, la implementación de mecanismos de participación ciudadana ha tenido un importante auge desde hace alrededor de tres décadas, en las que el continente ha presenciado la proliferación de una amplia variedad de dispositivos participativos en diversos ámbitos de la 
gestión de lo público, como los mecanismos de planificación y presupuestación participativa surgidos en la prefectura de Porto Alegre en Brasil y han sido replicado a lo largo y ancho de la región.

En el Ecuador, la puesta en marcha de espacios de participación se produjo desde la iniciativa de los gobiernos locales, a fines del siglo pasado, logrando impactos importantes en varios municipios comandados por autoridades indígenas que promovieron formas alternativas de administración participativa, en que los procesos de planificación tomaron mucha fuerza y alcanzaron avances importantes en términos de desarrollo local. Destacándose varias experiencias a nivel de la Sierra centro y norte, como el caso de Cotacachi.

Diversos estudios sobre la experiencia participativa de Cotacachi (Ospina, 2011; Ramírez y Espinosa, 2012; Ortiz, 2012), concuerdan en señalar que uno de los más ostensibles logros del proceso de participación de Cotacachi fue el impulso al surgimiento de nuevas organizaciones sociales que se integraron al denso tejido social del cantón, además de provocar un proceso de ciudadanización que generó una sociedad civil con una amplia formación en diversos ámbitos de la participación, así como también con importantes conocimientos de diferentes ámbitos de la gestión pública, tornándoles autoridades no estatales sobre el campo de la participación. De ese modo el proceso de participación se desarrolló desde una estructura social y política que le trasmitió el impulso con el que pudo generar avances importantes en términos de desarrollo local. y que al ponerse en marcha el nuevo régimen de participación de la Constitución de 2008 en el cantón, demandarían ser parte protagónica y activa del mismo.

La planificación participativa local estaba planteada desde el proyecto de gobierno como un espacio para la ampliación de la democracia mediante la intervención de la ciudadanía en los procesos de definición de la ruta hacia el desarrollo que debe seguir la localidad sin embargo, en la práctica en lugar de ejecutarse como un medio de ampliación de la democracia, en Cotacachi se intentó implementar como un mecanismo político de exclusión de actores sociales no alineados. Lo cual, activó en las fuerzas sociales de la participación diversas maniobras de defensa de su legitimidad como actores mediante los cuales se había construido el proceso de participación del cantón. De ese modo, el espacio de la participación en la planificación se tornó en un campo de conflictividad en que actores estatales como el gobierno central y local, y actores sociales, implementaron diversos mecanismos y formas de poder en el interés de regir la participación. Surgiendo de ese modo el siguiente objetivo de investigación: Conocer de qué manera se produjo el gobierno de la participación ciudadana en el proceso de planificación local en Cotacachi.

\section{DESARROLLO}

\section{MATERIALES Y MÉTODOS}

El presente estudio fue desarrollado dentro del paradigma científico interpretativo y mediante una investigación de tipo cualitativo se efectuó un estudio de caso de la planificación participativa en el cantón Cotacachi, estableciendo como población a los miembros y dirigentes de las organizaciones sociales que se articulan al proceso de participación ciudadana a través de la Asamblea de Unidad Cantonal de Cotacachi AUCC.

La información de primera mano se levantó a través de observación no participante realizada en los diferentes espacios participativos del cantón durante el periodo 2017-2018, además de entrevistas a actores clave del proceso participativo; mientras que la información de secundaria fue recabada mediante revisión bibliográfica y documental de documentos oficiales de organismos 
César Gonzalo Alvear Morales, , ...

estatales y no gubernamentales, legislación de nivel nacional y local, los diferentes planes de desarrollo del cantón.

Para el estudio del gobierno de la planificación participativa en Cotacachi, se empleó la perspectiva analítica de la gubernamentalidad de Foucault (2004), que señala que el gobierno de diferentes ámbitos de la sociedad se produce desde el ejercicio de poder que diversos actores (estatales y sociales) realizan a través de dos tipos de maniobras de gobierno: la formación de campos discursivos mediante los cuales el Estado justifica su intervencion en diverentes espacios de la sociedad, y las técnicas de gobierno mediante las cuales los actores involucrados ejercen el poder de manera práctica (Rose \& Miller, 2010, p. 273).

El estudio de caso se desarrolló de manera histórico-descriptiva presentando, en primer lugar, las estrategias de gobierno que desde el Estado central se pusieorn en marcha en el nuevo régimen de planificación participativa; y en segundo lugar, en el nivel local, se detalla la evolución del proceso de planificación participativa en Cotacachi, y los conflictos entre gobierno local y actores sociales por prevalecer en el proceso de implementación del nuevo régimen participativo establecido por la Constitución de 2008.

Se seleccionó como caso de estudio a Cotacachi en razón de su trayectoria en el ámbito de la planificación participativa, al haber sido un referente de la gestión del desarrollo local, cuyos mecanismos de participación fueron modelo -junto con otros casos exitosos- para la construcción del régimen de planificación participativa de la Constitución de 2008 (Machado 2012, 16). Habiendo mantenido vigentes sus espacios participativos a lo largo de 24 años, Cotacachi es una pocas experiencias referente que todavía se mantiene existente; de ese modo en su proceso se expresan y conjugan las dinámicas de la planificación particpativas del antes y después de la Carta Magna de 2008.

El análisis del caso se realizó bajo el enfoque analítico de la gubernamentalidad de Michel Foucault (2004) que plantea al gobierno de los diferentes ámbitos de la sociedad -en este caso, la participación ciudadana en el proceso de planificación- como un dominio de estrategias, procedimientos y técnicas, por medio de los que diversas fuerzas tratan de llevar a cabo sus programas de modo que el poder puede ser ejercido por actores estatales y no estatales; por tanto, el gobierno deja de concebirse como un organismo jurídico-político comandado por una autoridad que ha recibido un mandato formal y puede ser ejercido por los actores que disponen de los medios y las capacidades a través de la cuales pueden ejercer el poder de manera práctica. Respecto de la metodología de un estudio de gobierno (Miller y Rose 2010, 281).

\section{RESULTADOS Y DISCUSIÓN}

\section{La narrativa del Estado sobre la planificación participativa}

Según Rose (2000, p.145) el gobierno de diferentes ambitos de la vida de la sociedad se realiza a través de narrativas, a través de las cuales el Estado establece un régimen de verdad dentro del cual se justifica su intervención en la sociedad; además de diverentes tecnologías de poder a través de las cuales se implementa el gobierno de manera práctica.

En el caso de la planificación, el proyecto político de Alianza País, propuso a la participación ciudadana como el mecanismo fundamental a través del cual los diferentes ámbitos de la gestión pública se democratizarían, abriendo sus puertas a la participación de la ciudadanía, la cual sería el motor de cambio revolucionario planteado por el proyecto de gobierno.

Según Freidenberg y Pachano (2016, p.190), el surgimiento del Movimiento País se dio en el contexto de desgaste del sistema de representación política que desde el retorno a la democracia en 1979 no había logrado atender a las demandas de la ciudadanía, llegando así al agotamiento del 
sistema de partidos sumado a los levantamientos populares que pusieron fin a tres gobiernos en 1997, 2000 y en el 2005, y que desgastaron la confianza de la población en las alternativas políticas tradicionales.

Bajo esas condiciones, entró Alianza País en el tablero político, con un discurso diferente que recogía en su retórica las proclamas de lucha en contra del neoliberalismo de los movimientos sociales y las demandas de cambios profundos del Estado. Así logró sintonizar con los colectivos sociales y la población, alineándose al discurso de reivindicación y convocando a la sociedad a ser actora activa del proceso revolucionario que podría fin al neoliberalismo y transformaría la estructura estatal, en la que la acción ciudadana seria el eje del cambio a través de una "democracia radical y participativa" (Alianza País, 2006), que promovería la directa intervención de la ciudadanía en la gestión de los temas de interés colectivo, entre ellos la planificación nacional y local, que desde el discurso del proyecto político se la planteaba en los siguientes términos: "Para construir esa Patria nueva necesitamos hacer en la actual coyuntura un gran esfuerzo nacional, orientado a cristalizar una verdadera revolución democrática. Necesitamos construir ya una democracia radicalmente participativa" (p.8).

Ya en el gobierno, se impulsó la retórica de la participación en una instancia técnica como es la planificación, expresándola a través de un discurso que otorgaba un rostro social a la intervención de la ciudadanía en el desarrollo de la planificación, desde Senplades se la proponía de la siguiente manera:

La finalidad de la Participación Ciudadana en la planificación pública, es promover la intervención ciudadana en la formulación, implementación y evaluación de los diversos planes sectoriales y territoriales (...) Da lugar a la asunción social de responsabilidades públicas, conjuntamente con el ejercicio de los derechos ciudadanos encaminados al fortalecimiento de la cogestión pública. (Senplades, 2012, p.3)

De ese modo, se determinó a la planificación como la herramienta más importante para alcanzar el desarrollo, complementada con la participación como principio básico del Estado planificador que se instauró con la Constitución de 2008.

Una peculiaridad del discurso político estatal en el ámbito de la participación en la planificación es que tomó una configuración diferente a las narrativas que el Estado promovía en el resto de ámbitos de la gestión de lo público, en el sentido de que mientras en su discurso difundía en todos los ámbitos estatales la imagen de un gobierno altamente técnico, integrado por especialistas y técnicos que gestionarían la institucionalidad pública en los más altos estándares de eficacia y eficiencia ; mientras que en retórica implementada en el campo de la planificación que es un área eminentemente técnica y especializada, desde el discurso estatal se subordinaba a los funcionarios técnicos y se elevaba la valía de la ciudadanía en los siguientes términos:

Tradicionalmente las experiencias de planificación nacional han sido un proceso de especialistas y funcionarios públicos. El Gobierno Nacional se ha comprometido a impulsar un amplio proceso de participación social, en el que no hay cabida para una planificación confiscada por especialistas y expertos. (Senplades 2007, p.76)

Ya para el año 2013, el discursos estatal sobre la participación, planteaba la acción ciudadana, no como el motor de la revolución, sino más bien como una instancia promovida desde el estado, en ese sentido, desde el Plan Nacional del Buen Vivir 2013-2017 se señalaba que:

El principal agente de acción colectiva es, sin lugar a dudas, el Estado; pero no es el único. El gobierno ecuatoriano busca recuperar el Estado para la ciudadanía y, también, fomentar la acción 
César Gonzalo Alvear Morales, , ...

colectiva de la propia sociedad. Se parte del respeto a la autonomía de las organizaciones sociales y se reconoce el papel del Estado para promover la participación social y ciudadana. (Senplades, 2013, p. 80)

A través de toda esta narrativa, implementada como dispositivos intelectuales para la generación de campos de verdad, se procuraba desde el Estado la producción de un régimen de verdad en cuanto a la planificación y la participación, con la finalidad de conformar en ellas un campo gobernable por parte del Estado (Rose, 2000, p.145).

\section{Técnicas estatales de gobierno de la planificación}

Técnicas de gobierno multinivel

Desde la perspectiva de gubernamentalidad de Foucault (2004) el gobierno se realiza a través de medios técnicos expresados en tecnologías producidas desde los centros políticos, a través de las cuales se operativiza el poder del Estado. En ese sentido, para el gobierno de la planificación participativa, desde la Constitución se estableció la conducción de la misma a través de lo Paul Dugdale (2017) denomina como un régimen de gobierno multinivel, en el que a través de la articulación y la coordinación entre diferentes niveles de gobierno, se dirigen diversos ámbitos de la sociedad, de esa manera la participación en los espacios de planificación se estableció gobernarse a manera de un sistema articulado y coordinado.

Para ese fin se constituyó un Sistema Nacional de Planificación que mediante un conjunto de mecanismos técnicos dirige de forma bidireccional tanto la planificación como la participación ciudadana que en el proceso planificador se produce.

Bajo esa lógica se dispuso al Plan Nacional de Desarrollo como la columna vertebral a la cual debe anclarse obligatoriamente la planificación participativa de todos los niveles de gobierno autónomo descentralizado, ejecutando lo que Miller \& Rose (2010) señalan como un gobierno a distancia. El sistema conecta y articula los procesos de elaboración de los planes de los gobiernos autónomos descentralizados, y la variedad de planes y programas de carácter sectorial elaborados desde las diferentes carteras de Estado a la planificación del Estado central; disponiendo además la permanente producción y conocimientos para el continuo mejoramiento de los mecanismos técnicos de la planificación en las diferentes entidades de gobierno; la realización de actividades de seguimiento y evaluación de la puesta en marcha de lo planificado por las diversas instituciones de Estado, y el establecimiento de las directrices respecto de las cuales definir la inversión pública. Y fue precisamente en el ámbito de la planificación que el gobierno de la Revolución Ciudadana dio inicio a la participación en el año 2007 en el proceso de elaboración del Plan Nacional de Desarrollo 2007-2010, implementando la participación a través de talleres de consulta sectoriales en varias etapas de la elaboración del plan, en los que la ciudadanía fue convocada a participar dar sus aportes en el ámbito de diseño de la política pública y en la priorización de los ámbitos de intervención estatal en base a una serie de objetivos de desarrollo, en 69 mesas de consulta, en diferentes ciudades del país (Orrantia, 2018, p. 58)

En relación a la institucionalidad surgida para el gobierno de la planificación, se constituyó la Secretaría Nacional de Planificación Senplades como el organismo rector de la planificación en todo nivel de gobierno, además que, por disposición del Reglamento del Código Orgánico de Planificación y Finanzas Públicas: sería la entidad a cargo del diseño de los instrumentos y técnicas para la puesta en operación del Sistema Nacional de Planificación (Art.5).

Mientras que para el gobierno de la participación en el nivel local se estableció desde la Constitución y las leyes, a los gobiernos autónomos descentralizados como la autoridad sobre el 
ámbito de la planificación y de la participación que se debe realizar en su elaboración. De ese modo, el gobierno multinivel es de la participación quedó conformado por la articulación de instituciones del gobierno central y del régimen autónomo descentralizado.

\section{El plan de desarrollo y ordenamiento territorial PDOT}

De entre los diversos mecanismos que fueron diseñados y puestos en vigencia para el gobierno de la planificación, se establecieron para el nivel local el proceso de elaboración del plan de desarrollo y ordenamiento territorial. De acuerdo al Código Orgánico de Planificación y Finanzas Públicas, el PDOT es la herramienta principal en la gestión del desarrollo en las circunscripciones territoriales cantonales, a través del cual se orienta la intervención del gobierno local en el territorio (Art.41).

Mediante disposición constitucional, el PDOT debe realizarse con la participación de la población (Art.100), señalando la Secretaría Técnica Planifica Ecuador, que la participación debe implementarse en el desarrollo de sus tres grandes componentes: el diagnóstico que describe y analiza la situación del territorio en cuanto a inequidades; la propuesta, que recoge y expresa la visión del cantón en el mediano plazo, así como los objetivos a seguir además de las metas, programas y el modelo territorial deseado; y finalmente el modelo de gestión en el que se trazan las estrategias por medio de las cuales se plantea alcanzar lo planeado.

La elaboración de los diferentes componentes del PDOT es llevada a cabo por un equipo técnico, mientras que la participación de la ciudadanía se produce mediante mesas de trabajo en las que se definen los lineamientos de los diferentes ámbitos que integra el plan, estos son recogidos por el equipo técnico y en función de ellos desarrollan los componentes del plan.

El proceso de elaboración del plan de desarrollo y ordenamiento territorial es un procedimiento de alto contenido técnico que involucra que su construcción sea llevada por especialistas de diferentes materias; por tanto, los espacios de participación que se generan para la elaboración del plan requieren en la ciudadanía un acervo de información, conocimientos y criterios para la comprensión de las diferentes temáticas trabajadas, así como también para realizar propuestas; sin embargo, no es frecuente ese nivel de conocimiento y formación en la ciudadanía, salvo en algunos temas muy puntuales de manera que la participación de la gente se ve reducida a aportes bastante generales.

De esa manera se puede percibir cómo el espacio de participación en la planificación, aunque es abierto para la intervención de la ciudadanía, en la práctica funcionaría como un ámbito especializado que demanda en la población un importante nivel de preparación que al no tenerlo su participación se vería reducida, no por maniobras de exclusión de las autoridades, sino por la limitación de conocimiento que podría tener la ciudanía. Produciéndose así un modo de participación que Velásquez y González (2003) señalan como "participación-integracióncooptación" (p.23) en la que la autoridad del gobierno local promueve la participación a través de la creación de nuevos espacios para la intervención de la ciudadanía, convocando a la población a involucrarse en ellos, haciendo uso permanente del discurso de la participación enfocado a consolidar las orientaciones de los grupos políticos dominantes antes que promover en la ciudadanía mayor capacidad de autonomía y de interlocución con el Estado.

De esa manera se puede ver en el gobierno de la participación insertada en los procesos de planificación lo que señalan Rose y Miller (2010) en torno a "que el poder no se trata de imponer restricciones" (p.272) pues en el caso del campo de la planificación participativa, el espacio se abre para la intervención de la ciudadanía en el establecimiento de las líneas de desarrollo hacia las que el ciudadano aspira que la intervención del gobierno local se oriente; sin embargo, al no tener la formación técnica para una participación efectiva su intervención no logra trascender. De ese modo, el gobierno de la planificación participativa, se plantea como un dominio de conocimientos. 
César Gonzalo Alvear Morales, , ...

\section{La planificación participativa en Cotacachi}

La experiencia de participación de Cotacachi nace precisamente con la puesta en marcha de un proceso de planificación del desarrollo local impulsado desde la alcaldía en 1996, encaminado a la elaboración del primer plan cantonal y uno de los primeros de nivel local en el país. dos años antes de la promulgación de la Constitución de 1998, dándose inicio al proceso de participación cuando el derecho de la participación no era todavía reconocido; sustentando el proceso participativo en el acuerdo entre la autoridad y la sociedad civil que encontró en la participación un mecanismo a través del cual ser escuchada y lograr que sus necesidades sean atendidas, sus derechos alcanzados, y sus condiciones de exclusión sean superadas, volviéndola al mismo una acción permanente, de ese modo la participación en el cantón se tornó un "proceso vivo (...) que surge de la propia reproducción social de la vida" (Unda, 2013, p.252-253).

El Plan de Desarrollo Cantonal de Cotacachi abrió las puertas en el cantón para la intervención de la ciudadanía en un territorio marcado por la inequidad y varios índices de desarrollo ubicados entre los más bajos del país, sobre todo en las zonas rurales en donde poblaciones como la indígena habían logrado sus derechos fundamentales apenas hace un par de décadas.

En esas condiciones se impulsó el proceso de planificación: creando espacios para la expresión de las demandas de los diversos grupos humanos de las diferentes zonas del cantón, quienes encontraron en la participación una vía por la cual podrían canalizar sus requerimientos y reafirmar la identidad de sus territorios.

Según varios estudios, Cotacachi es un cantón con una tradición organizativa de larga data, conformando a nivel de sus tres zonas una amplia y consolidada red de organizaciones ligadas profundamente con los procesos territoriales de construcción de condiciones de mayor equidad e inclusión como es el caso de las comunidades indígenas de la zona Andina del cantón que a través de la organización canalizaron sus luchas contra el racismo y la explotación .

Estas organizaciones desde sus formas de lucha aportaron la base social y la trayectoria de participación popular y comunitaria que asumió el proceso participativo de Cotacachi, así como también el colectivo de organizaciones de defensa de la naturaleza de la zona de Intag, que a través de la resistencia ambientalista contra los intentos de empresas multinacionales mineras y del propio Estado de llevar a cabo la explotación minera de los yacimientos de cobre de zona, logrando contener los intentos de explotación en sus territorios .

Según el Plan de Desarrollo Cantonal de Cotacachi 1997, para su elaboración del plan se llevaron a cabo diez talleres participativos en las zonas: Andina, Urbana y de Intag, cuatro encuentros interculturales, además de dos foros ambientales en la zona subtropical y en la zona Andina (PNDC, 1997). De esa manera la planificación participativa en el cantón se alimentó de los aportes de diversos sectores y de diferentes experiencias de lucha por derechos y diversas líneas de pensamiento y acción sobre el desarrollo, como el caso de las organizaciones indígenas y del colectivo inteño identificados con importantes líneas del plan como: seguridad alimentarias, medicina tradicional, medio ambiente, salud; hacia las que se orientaron los esfuerzos y capacidades de las organizaciones y territorios para alcanzar la metas planteadas y respaldar las estrategias acordadas en conjunto con las autoridades (Ospina, 2011). A ellos se sumaron los grupos organizados de la zona urbana que en el ámbito productivo se articularon los artesanos para dar impulsar las estrategias de fomento turístico. Así la participación de los actores sociales se sumó al proceso de planificación no solo en la definición de las directrices del desarrollo sino en su posterior puesta en marcha en cada uno de los territorios, asumiendo una concepción de la 
participación más allá de las mesas de consulta y los talleres, e insertándolos en los objetivos del territorio hacia los cuales se encausaría la acción de las comunidades y la diversidad de organizaciones. Elaborado el plan, la sociedad civil se empoderó del proceso de implementación, provocando la multiplicación de instancias de ejecución y seguimiento de carácter sectorial conformadas por consejos integrados por representantes de las organizaciones sociales, la Asamblea de Unidad Cantonal, y autoridades del gobierno local, en los ámbitos de: educación, gestión ambiental y manejo de recursos naturales, producción agropecuaria, producción artesanal y salud; encargados de dar seguimiento a los diferentes componentes del plan, además de la ejecución de los programas y proyectos a través de cogestión con el gobierno local o agencias de cooperación internacional.

Así la planificación participativa escaló hacia otro nivel en la experiencia de Cotacachi al desarrollarse procesos de micro planificación participativa a nivel de los consejos sectoriales, que llegaron a ejecutar programas de mucha trascendencia como el programa de alfabetización "Yo sí puedo- Ñuka Ushanimi" promovido por el Consejo intersectorial de Educación con el que el cantón pudo reducir sus índices analfabetismo de 22,3\% a 3,4\% (Arboleda, 2006, p.170), o el proceso descentralización de la competencia de salud impulsado por el Consejo de Salud, mediante el cual Cotacachi implementó su propio sistema de salud.

Adicionalmente la planificación participativa se ramificó hacia otros ámbitos, sectores y organizaciones como el Plan de Vialidad Cantonal, el Plan de Desarrollo de la Unión de Organizaciones Indígenas y Campesinas de Cotacachi UNORCAC, el Plan de Niños y Adolescentes, el Plan Cantonal de Salud que sumados superaban las dos decenas de procesos de planificación participativa (Cevallos, 2015).

Cabe mencionar que todo el desarrollo que tuvo la planificación participativa en el cantón no se produjo bajo el marco de una ley que respalde el proceso, sino tan solo se sustentó en el acuerdo entre los actores del territorio, además de el empoderamiento de las organizaciones respecto del proceso, otorgándole el respaldo político y social necesario para poder materializar los objetivos construidos colectivamente. De ese modo, la participación en Cotacachi surgió basada en la convicción de que la legitimidad del proceso la daba la sociedad civil antes que determinaciones legales, lo cual reforzó la concepción de una participación autónoma.

A través de este proceso, la sociedad civil organizada y la Asamblea de Unidad Cantonal ganaron una amplia experiencia, capacidad técnica y conocimientos en la planificación del desarrollo local. Este acopió de destrezas, provocó que los debates a nivel de los espacios de participación tuviesen un nivel cada vez más alto, de modo que las autoridades y los técnicos del gobierno local ya dejaron de ser los únicos con el conocimiento técnico y especializado en el campo de la planificación, y viendo disputado su protagonismo con la sociedad civil, señal la primera presidenta de la AUCC que el alcalde empezó a recelar a los actores locales que habían desarrollado todo su potencial y figuraban como actores políticos de alto rendimiento.

Por tanto, el alcalde que dio inicio al proceso participativo empezó a marcar distancias de manera progresiva hasta separarse completamente del proceso y romper relaciones con la Asamblea de Unidad Cantonal y el proceso de participación, ante lo cual, las organizaciones y la AUCC, asumieron el liderazgo de la participación en Cotacachi.

De modo que al llegar las disposiciones de la Constitución de 2008 sobre la implementación de la planificación participativa al cantón, en este ya se hallaba desde hace más de una década y media un régimen de gobierno civil de la participación consolidado en el tejido social del cantón y en su estructura política a través del poder de la participación que se había generado desde el proceso participativo. Por tanto, desde los actores sociales y la AUCC se demandaría protagonismo en la implementación del nuevo régimen constitucional de la planificación participativa que tenía que impulsar el gobierno local. 
César Gonzalo Alvear Morales, , ...

La puesta en marcha de la planificación participativa de la Constitución de 2008 en Cotacachi. En el año 2009, asumió el gobierno local un histórico dirigente indígena: Alberto Anrango, que bajo la bandera política de Alianza País sería quien tendría que inaugurar los diferentes espacios participativos establecidos por la Constitución.

El peso político de la línea partidista de gobierno iría ejerciendo presión sobre el alcalde en cuanto a establecer nuevos espacios de participación sin la presencia e influencia de las organizaciones sociales y la AUCC. Según Unda (2013) la línea política del gobierno de Rafael Correa Delgado respondía a un proyecto político hegemónico que buscaba acumular poder sobre los diferentes espacios en detrimento de los actores sociales a quienes el proyecto político intentaba sujetar a los lineamientos del Ejecutivo; de modo que los nuevos espacios de participación en la planificación se miraban como la oportunidad de conformar un nuevo campo hacia donde extender el poder del oficialismo, a la vez de debilitar la presencia que los actores de la participación llegaron a tener en el cantón.

En ese contexto, la relación del gobierno local con las organizaciones y la AUCC había escalado en tensión a razón de la determinación municipal no dar cabida en los nuevos espacios de planificación participativa a actores sociales importantes, así como a la Asamblea de Unidad Cantonal, desconociendo su legitimidad. Produciéndose lo que (Merry, 2001, p.19) denomina como "técnicas de gubernamentalidad espacial" a través de las cuales la autoridad conforma nuevos espacios de gobierno -en este caso de gobierno de la participación- a través de maniobras destinadas a establecer el control social mediante la exclusión de individuos o grupos considerados como un riesgo para el orden ciudadano dentro de esos nuevos espacios de gobierno.

Sin embargo, por sobre la intensión municipal de dejar fuera al proceso participativo de la sociedad civil organizada, se encontraban las capacidades municipales de poner en marcha procesos de planificación participativa totalmente nuevos y en los que no tenía la experticia y los conocimientos especializados, poniendo en evidencia lo que señala Michel Foucault (2004) que las maneras en las que se produce el gobierno se hallan basadas en conocimiento experto, el cual en el ámbito participativo de Cotacachi se encontraba de lado de los actores sociales y la Asamblea de Unidad Cantonal, además que el proceso de planificación participativa involucraba la movilización y participación de una diversidad de grupos que se encontraban insertados en el proceso participativo de las organizaciones y la AUCC.

De ese modo, el régimen de participacion establecido en Cotacachi y regido por un gobierno civil de la misma, establecía una estructura política muy difícil de excluir. Por tanto, implementación de la planificación significó un desafío de magnitud para el Municipio en cuanto a sus propias capacidades para ponerlo en marcha y las capacidades de los actores sociales para hacer valer la legitimidad ganada, así como sus derechos de participación.

Esta primera elaboración del PDOT de manera participativa establecido para los GAD de todo el país un reto de mucha exigencia en el hecho de que la mayoría de autoridades y equipos técnicos municipales no poseían experiencia en procesos y metodologías de planificación participativa. A eso se sumó el establecimiento del Gobierno central de una fecha límite para la entrega de los PDOT cuyo incumplimiento involucraría que el Ministerio de Finanzas no proceda a entregar las trasferencias económicas que los GAD reciben del Gobierno central y que involucran la mayor parte de ingresos que disponen los gobiernos locales para su operación. La fecha límite de entrega establecida fue el 31 de diciembre de 2011.

Según datos de la Asociación de Municipalidades Ecuatorianas citados por la Senplades (2013), a noviembre del año 2012, cuarenta de los 221 cantones del país no disponían de un plan de 
desarrollo y ordenamiento territorial, de modo que alrededor del $80 \%$ de municipios no lograron realizar sus PDOT.

En el caso de Cotacachi, según la presidenta de la AUCC del periodo 2009-2013, la intención municipal de dejar fuera del proceso de planificación participativa a las organizaciones sociales que no eran de su agrado además de la Asamblea de Unidad Cantonal, se encontró con la dificultad de que para poner en marcha procesos de participación en lugares con una estructura de participación consolidada como Cotacachi, no era suficiente con tener el conocimiento y manejo de las metodologías, lo cual se hubiese resuelto contratando un experto, ya que además del saber demandaba la coordinación de una ampla variedad de actores territoriales y sectoriales del cantón que ya se hallaban vinculados al proceso participativo de la AUCC, a lo cual se sumaba la carrera contrarreloj que debía efectuar el Municipio para lograr culminar todo el proceso dentro de las fechas límite impuestas por el Gobierno central.

Ante el cúmulo de dificultades presentadas para llevar a cabo el proceso de planificación, el gobierno local dio paso a la integración de la Asamblea de Unidad Cantonal con todos los actores sociales al proceso planificador, produciéndose el reconocimiento tácito del Municipio a la legitimidad de las organizaciones sociales y a la AUCC, quienes al sumarse a la elaboración del PDOT articularon esfuerzos con el equipo municipal y la participación empezó a fluir hacia la elaboración del PDOT.

En las mesas de trabajo de los espacios participativos, el nivel de manejo de las temáticas de los actores sociales provocó que desde ellos se impulsen las agendas de cada territorio, provocando intensos debates con el equipo municipal que, según la presidenta de la AUCC del periodo, intentaban promover los lineamientos de la política del Gobierno central en temas como el ambiental en que desde el Ejecutivo se quería dar el impulso a la explotación minera en la zona subtropical de Intag, lo cual chocaba con los planteamientos de defensa de la naturaleza que establecieron como la base de lucha de las organizaciones de la zona.

Pese a los sólidos argumentos legales del equipo municipal para justificar el no acoger las demandas ambientalistas de las organizaciones de Intag, el desenvolvimiento y dominio del tema por parte de los miembros y dirigentes del colectivo ambientalista logró prevalecer sobre las determinaciones extractivistas que se intentaban implementar en el plan, logrando por parte de los actores sociales la ratificación de Cotacachi como un "Canton Ecológico", quedando establecido en la visión que en el cantón el ámbito productivo se regiría bajo criterios de sostenibilidad, solidaridad, no extractivista y respetuoso con la biodiversidad.

Como requerimiento para la aprobación del PDOT, se establecía que la aprobación del mismo por parte de algunas instancias, entre ellas el sistema de participación cantonal, entidad parte del nuevo régimen de participación establecido por la Constitución y que desde los actores sociales se demandaba al gobierno local el reconocimiento a la AUCC como el sistema de participación, en razón de que desde el inicio del proceso participativo en 1996 había funcionado como el sistema de participación cantonal.

No obstante, la determinación municipal era la de conformar otro sistema de participación afín a la linea política del gobierno local; sin embargo, la creación de un nuevo sistema de participación paralelo al existente en la AUCC y sus organizaciones, fue una tarea que no logró sacar adelante el Municipio, por lo que, una vez finalizado el PDOT, se vio obligado a reconconocer a la Asamblea de Unidad Cantonal como el sistema de participación cantonal de manera parcial, mediante la Ordenanza de Aprobación del Plan de Desarrollo y Ordenanmiento Territorial del Cantón Santa Ana de Cotacachi el 15 de agosto de 2011.

De acuerdo a Foucault (2004), el gobierno va más allá de una autoridad delegada; involucra las instituciones, cálculos, tácticas, conocimientos y reflexiones que permiten la materialización del 
poder. En el caso la planificación en Cotacachi, el Municipio para poder gobernar la participación en el proceso de planificación, tenía a su favor la autoridad delegada por la Constitución; sin embargo, no disponía de la institución participativa consolidada en el territorio como la AUCC, las fuerzas sociales que deben articularse en toro al proceso de planificación, los conocimientos, las tácticas y tecnologías participativas que, en cambio, sí disponían los actores sociales y la Asamblea de Unidad Cantonal y que les permitieron el ejercicio de poder en la pugna producida con el Municipio. De esa manera se ratificaba que la participación en Cotacachi se mantenía bajo un régimen de gobierno civil, al que el Municipio intentó eludir sin éxito.

La planificación en Cotacachi en el preriodo post transición constitucional

Para las elecciones seccionales del año 2014, transcurrido ya el periodo de transición en el que debían ponerse en marcha los mecanismos participativos determinados por la Constitución, algunas intancias de la participación local todavía estaban sin definir en el sentido de que el Municipio no logró conformarlas mediante el establecimiento de un sistema de participación local.

Bajo esas circunstancias, las organizaciones de la participación en Cotacachi, optaron por la vía de la democracia reprentativa como el camino a través del cual consolidar su proceso de particiapción desde el reconocimiento municipal, y para el efecto articularon el capital político acumulado a través de la participación en las diferentes zonas del cantón, además de todas las capacidades organizacionales y tecnicas ganadas en el proceso participativo, y las canalizaron hacia la creación de un movimiento politico propio que reivindicaba las demandas de las organizaciones de la particiaciòn reconociéndoles su legitimidad y poniendo en valor su trayectoria de casi dos decadas. De ese modo se constituyó el movimiento político de las organizaciones, el cual logró alcanzar el poder municipal con un candidato propio proveniente del proceso de participación. Una vez en el poder, se puso en marcha un proceso de construcción participativa de los que sería el nuevo sistema de participacion y sus diferentes instnacias como la planificación participativa. Culminado el proceso se reconoció desde el Municipio a la Asamblea de Unidad Cantonal como sistema de participacion local, así como sus instancias y a las organizaciones articuladas a ella, a través de la promulgació de la Ordenanza del Sistema de Participación del Cantón Santa Ana de Cotacachi "Asamblea de Unidad Cantonal de Cotacachi" del 30 de septiembre de 2016, mediante la cual en el proceso de planificación local se estableció como una instancia de de articulación y coordinación de la AUCC, las oganizaciones y el Municipio.

Con el inicio del nuevo periodo municipal, tenía que llevarse a cabo la actualización del PDOT del cantón, el cual se produjo mediante la coordinación entre los actores sociales, Municipio y AUCC, a través de procesos particiapativos ejecutados en los diferentes espacios del cantón y un conjunto de mesas sectoriales, a través de los que se ratificó los lineamientos de desarrollo que establecidos desde cada zona etno geográfica del cantón, corroborando el principio de defensa de la naturaleza en el cantón en contra de la explotación minera en Intag, señalando a Cotacachi como un cantón ecológico y enfocado en un desarrollo participativo, con enfoque de género, intercultural, generalcional y ambiental.

De esa manera, desde las oganizaciones sociales y desde la AUCC se logró mantener en vigencia el proceso particiapativo de más de dos decadas y ratificar el gobierno civil de la participación que los actores sociales y la Asamblea de Unidad Cantonal sobre la particiación ciudadana en el cantón.

\section{CONCLUSIONES}

En términos generales, se estableció el gobierno de la participación en el proceso de planificación desde tres instancias: el Gobierno central, el gobierno local y los actores sociales del territorio. Cada uno rigiendo diferentes esferas del ámbito de la planificación participativa local. 
Desde el nivel nacional se estableció para el gobierno de la participación en la planificación dos tecnologías de gobierno: un régimen de gubernamentalidad multinivel y un campo de narrativas estatales.

En el caso de la gubernamentalidad multinivel, desde el Estado se la implementó estableciendo dos ámbitos de gobierno de la participación en la planificación: uno político y uno técnico. En el ámbito político se estableció la delegación de una autoridad específica desde la Constitución a cada nivel de gobierno para regir sobre la planificación y por ende a los espacios participativos que se producen al interior de los procesos.

En lo técnico, se implementó a través de instituir un organismo técnico rector sobre el ejercicio de la competencia de planificación, desde el cual se definían los lineamientos técnicos, formatos y procedimientos a través de los cuales se implementa la planificación y la participación; mientras que a los gad se les determina la operativización del instrumental técnico.

Desde esa distribución de autoridad y funciones, a la ciudadanía se le asigna un rol consultivo; sin embargo, en Cotacachi la sociedad civil desarrolló capacidad técnica y capital político en el ámbito participativo, mediante los cuales su rol en el proceso de planificación no se sujetó a ser un ente consultivo, sino un actor protagónico ejerciendo un poder no proveniente de una determinación legal, sino de una construcción social del poder ciudadano en el espacio de participación.

Para justificar ante la población la institucionalización de la participación en el proceso de planificación local, la cual apuntaba a ser un espacio para la ciudadanía administrado por el Estado, se estructuró una retórica que apuntaba al protagonismo que la población como eje del proceso de planificación, a cuya intervención se subordinaría la gestión técnica de los equipos de especialistas que llevan el proceso, así como también la autoridad del gobierno local que se acogería; de modo que desde la participación de los grandes levantamientos que se produjeron en contra del neoliberalismo y del desgatado sistema político se canalizaría a otra forma de incidencia en los espacios de participación establecidos para sujetarse a los dictámenes del poder popular; sin embargo en la operativización de la participación fue configurada como espacio de consulta gobernado por la autoridad local y sus equipos de técnicos, ya que el ámbito de la planificación es un espacio regido en base a conocimiento especializado y experticia en los instrumentos y metodologías participativas y de planificación; que en el caso de Cotacachi, la sociedad poseía el conocimiento y la destreza técnica y política, que le permitió prevalecer como un actor determinante en el proceso de construcción del PDOT.

A nivel local, en Cotacachi, el proceso participativo iniciado en 1996 que contemplaba todo un proceso de planificación que involucró además la ejecución con la intervención de las organizaciones sociales, otorgó a la sociedad civil del cantón una serie de capacidades, así como un capital político que volvieron a los actores sociales autoridades no estatales de carácter técnico y político sobre los diversos espacios de participación. De ese modo, se produjo lo que (Miller \& Rose, 2010, p.277) señalan como la existencia de múltiples autoridades sobre diversos ámbitos de la sociedad, y en el caso de la participación en Cotacachi, los actores sociales al acopiar conocimientos, capacidades y experticia se establecieron como autoridades civiles de la participación, sin embargo, no fueron la única autoridad, ya que al GAD se le otorgó una autoridad legal sobre la planificación al recibir la competencia que la Constitución le otorgó.

\section{REFERÉNCIAS BIBLIOGRÁFICAS}

Alianza País. (2006). Plan de Gobierno del Movimiento Alianza País 2007-2011. Recuperado el 11 de Diciembre de 2019, de https://izquierdaporelmundo.files.wordpress.com/2015/11/manifiesto_ideologico_ap.pdf

Arboleda, M. (2006). Género y gobernanza territorial en Cotacachi y Cotopaxi. En P. Ospina (Ed.). Instituto de Estudios Ecuatorianos. 
Cevallos, J. (2015). Experiencias de Asambleas Nacionales. Experiencia de Cotacachi. Primer Encuentro Nacional de Participación. Cotacachi.

Creswell, J. W. (2013). Research Design: Qualitative, Quantitative and Mixed Methods. SAGE.

De la Torre, C. (2013). El tecnopopulismo de Rafael Correa ¿Es compatible el carisma con la tecnocracia? Latin American Research Review, 24-43.

Dugdale, P. (2017). Multi-Level Governmentality. En D. Katherine, \& A. Kay (Edits.), Multi-Level Governance: Conceptual Challenges and Case Studies from Australia (págs. 101-120). Autralia: ANU Press.

Foucault, M. (2004). Securité, territoire, population. En Cours au Collège de France 1975-1976. Paris: Seuil Gallimard.

Freidenberg, F., \& Pachano, S. (2016). El sistema político ecuatoriano. Quito, Ecuador : FLACSO Ecuador. Obtenido de http://www.simonpachano.com/uploads/2/1/4/3/21439124/freidenberg-pachano_el_sistema_politico.pdf

López, M. (2011). Entre la identidad y la ruptura territorial: la construcción socio-histórica y socio-económica en Intag. Quito: Flacsoandes.

Machado, D. (2012). ¿Una nueva etapa de los movimientos sociales en el Ecuador? (W. Suquillo, \& N. Rivas, Edits.) La Tendencia. Revista de análisis político(13), 14-24.

Merry, S. (2001). Spatial Governmentality and the New Urban Social Order: Controlling Gender Violence Through Law. American Anthropologist, New Series, 103, 16-29.

Miller, P., \& Rose, N. (2010). Political power beyond the State: problematics of goveernment. The British Journal of Sociology, 271-303.

Orrantia, D. (2018). La planificación participativa en la elaboración del Plan Nacional del Buen Vivir 2013-2017. Quito: Universidad Andina Simón Bolívar.

Ortiz, S. (2012). Comuneros kichwas o ciudadanos ecuatorianos? La ciudadanía étnica y los derechos políticos de los indígenas de Otavalo y Cotacachi (1990-2009). Flacso-Ecuador.

Ospina, P. (2011). Borrador del libro Cotacachi (2008-2011) proceso participativo, crisis y oportunidades. Quito.

Ramírez, F., \& Espinosa, A. (2012). Ocupando la silla vacía: Representación, participación en el tránsito posconstitucional del Ecuador. Cuadernos del CENDES 29, 109-140.

Ramírez, F., \& Welp, Y. (2011). Presentaciòn del dossier: Nuevas instituciones participativas y democráticas en América Latina. Íconos: Revista de ciencias sociales, 40, 11-20.

Rose, N. (2000). Governing Liberty. En R. Ericson, \& N. Stehr (Edits.), Governing Modern Societies (págs. 141-176). University of Toronto Press.

Roy, A. (2009). Civil Governmentality: The Politics of Inclusion in Beirrut and Mumbai. Antipode, 159-179.

Senplades. (2007). Plan Nacional de Desarrollo 2007-2010. Recuperado el 5 de Julio de 2019, de https://www.planificacion.gob.ec/wp-content/uploads/downloads/2013/09/Plan-Nacional-Desarrollo-20072010.pdf

Senplades. (2012). La Participación Ciudadana para la Vida Democrática. Quito.

Senplades. (7 de Febrero de 2013). Boletín de prensa: Senplades realizó un taller de evaluación de la formulación de los Planes de Desarrollo y Ordenamiento Territorial. Recuperado el 3 de 10 de 2019, de https://www.planificacion.gob.ec/senplades-realizo-un-taller-de-evaluacion-de-la-formulacion-de-los-pdot/

Torres, V. H. (1999). El desarrollo local en el Ecuador. En G. D. Local, Ciudadanías Emergentes: Experiencias democráticas de desarrollo local (págs. 15-38). Quito: Abya Yala.

Unda, M. (2013). Las tensiones actuales de la participación. En C. Viteri (Ed.), Participación ciudadana, políticas públicas y educación (págs. 249-274). Quito: Contrato Social por la Educación, Organización de Estados Iberoamericanos - Oficina Ecuador, Educación Sin Fronteras - Ecuador.

Velásquez, F., \& González, E. (2003). ¿Qué ha pasado con la participación ciudadana en Colombia? Bogotá. 